\title{
P-18: Comparison of Lateral Abdominal Muscle Thickness in Young Male Soccer Players With and Without Low Back Pain
}

\author{
Bahareh Tavana ${ }^{1}$, Pardis Noormohammadpour ${ }^{1}$, Shadi Mirzaei ${ }^{1}$, Navid Moghaddam ${ }^{5}$, \\ Mohammad Ali Mansournia ${ }^{3}$, Masud Yunesian ${ }^{4}$, Ramin Ramin Kordi ${ }^{2}$ \\ ${ }^{1}$ Department of Sports and Exercise Medicine, School of Medicine, Tehran University of Medical Sciences, \\ Tehran, Iran Medical Sciences, Tehran, Iran \\ ${ }^{2}$ Sports Medicine Research Center, Neuroscience Institute, Tehran University of Medical Sciences, Tehran, \\ Iran \\ ${ }^{3}$ Department of Epidemiology and Biostatistics, School of Public Health, Tehran University of Medical \\ Sciences, Tehran, Iran \\ ${ }^{4}$ Department of Environmental Health, School of Public Health, Tehran University of Medical Sciences, \\ Tehran, Iran \\ ${ }^{5}$ Rasoul Akram Hospital, Iran University of Medical Science, Tehran, Iran
}

\begin{abstract}
PURPOSE: To compare the lateral abdominal muscle thickness and other possible functional risk factors in young soccer players with and without low back pain (LBP).

METHOD: In total, 30 young soccer players between 16 and 20 years old, with and without LBP, from the premier league participated in this study. The thicknesses of external oblique, internal oblique and transversus abdominis on both sides were measured via ultrasound imaging. In addition, hamstring flexibility, active lumbar forward flexion, and isometric muscle endurance of trunk extensors were measured and were compared regarding the history of LBP.

RESULTS: Mean \pm SD age of the subjects was $17.4 \pm 1.1$ years. There was no statistically significant difference regarding age, BMI, weekly training hours and age of starting to compete between groups. Subjects with sports-life, last year and last month history of LBP had a statistically significant lower external oblique muscle thickness in both right and left side, and both dominant and non-dominant feet $(p<0.05)$. Subjects with sportslife history of LBP had lower internal oblique muscle thickness in both side and both feet $(p<0.05)$. Moreover, those with a sports-life history of LBP had a significantly higher degree of hamstring muscle tightness than non-LBP group on the dominant foot $(p<0.05)$.

CONCLUSION: In this sample group of young soccer players, abdominal muscles seem to have an important role in the stability of the spine and prevention of LBP. Further longitudinal studies are needed to evaluate the role of these muscles as a risk factor for soccer players.
\end{abstract}

Available at: http://journalofsportsmedicine.org and http://dx.doi.org/10.5152/tjsm.2017.065

(C2017 Türkiye Spor Hekimleri Derneği. Tüm hakları saklıdır. 